\title{
Research on Core Strength Training Practice in Basketball Sports Xiangyang Xie
}

Guangzhou Vocational Institute of Sport, Guangzhou, 510650, China

\begin{abstract}
Keywords: Basketball sports; core strength training; training practice
\end{abstract}
\begin{abstract}
The core strength training in basketball sports is an important and key training task and relates to many technical action levels. Therefore, the core strength should be trained specially and effectively in basketball sports, so as to make basketball players have solid body posture, do the coordination of their upper and lower limbs well and make their physical conditions reach the highest level, thus promoting the effective combination and coordinated development of each links of body and greatly improving the overall level in basketball sports. This paper will discuss the core strength training in basketball sports in detail, expecting to make the physical training of basketball players scientific, reasonable and high-efficient and finally improve the competition level and performance of Chinese basketball sports.
\end{abstract}

\section{Introduction}

With the development of modern scientific theory and technology, the core strength training in basketball sports becomes more and more important, which is one of the effective ways of making the physical training of basketball players scientific, reasonable and high-efficient. Actually, in basketball sports, many technical actions cannot leave the core strength training. The basketball players can keep the solid body posture in the basketball sports only when they have strong core strength. The core strength training can help basketball players solve the problem concerning the power transmission and generation of core areas or links in traditional strength training, make the body of players in a more coordinated state, and solve the problem concerning body imbalance in the original strength training, thus beneficial to improve the technical action completion level and ability of basketball players. Therefore, to further shorten the gap with the basketball players of developed countries all over the world, the basketball players should be of core strength training by using the scientific training method.

\section{Main connotation of core strength}

The core strength mainly refers to the core parts of human body, that is, lumbar-pelvis_-hip joint, which play a role of controlling the movement gravity center and transmitting the power of upper and lower limbs, so as to generate certain power. It is a power generation source in the human motion. From the perspective of anatomy, the core strength is the strength parallel to the upper and lower limbs strength. The core strength training greatly differs from the traditional strength training because it is a strength stability and balance training of the core muscle group and deep small muscles of the body that cannot be reached by the traditional strength training, thus making the players keep certain core stability in the movement. For example, in basketball sports, the players are required to have a strong physique and body to complete various defensive and offensive movements with proper gesture. As the core stability in basketball sports mainly depends on the strength and cooperation of muscle, ligament and connective tissue of core parts, so the core strength training is an inevitable requirements of core stability, which can help basketball players gain good movement power and improve their body balance and stability.

\section{Important functions of core strength training in basketball sports}

The core strength training is of important function in basketball sports, which can improve the technical action skills and level of basketball players, especially the shooting average and stability 
of basketball players. Therefore, the core strength training of basketball players is necessary and important because it does improve the performance of basketball players in competition and training. The important functions of core strength training are as follows:

(1) Body shape of basketball players. Generally, the players are required to be tall in basketball sports, which makes them have poor physical stability and slow speed in the basketball games or training while bringing them advantages in basketball sports. So they may lose certain balance in case of fierce competition and physical confrontation. Thus, the core strength training of players in basketball sports is beneficial to improve their stability and control ability. Actually, for tall basketball players, the core area is one of the important guarantees for their body control and stability in sports, which can balance and coordinate their power generation movement, reasonably and effectively transmit and distribute the body strength and help them always in a stable and coordinated state in quick movements. In addition, it can also help basketball players overcome the problem concerning the body flexibility and coordination caused by their tall stature and is beneficial to help them complete the high-quality basketball technical action.

(2) Different techniques of basketball players. In basketball sports, the core of the kinematic link of all technical actions is the central muscles, which play an important role in supporting the stability of technical actions of players. For example, when the player jumps for shooting, the working of his muscles is a periodic process from stretching to contraction and the player can keep a stable shooting state by the power generation of lower limbs and combining with the physical stability, so as to greatly improve the shooting average. Actually, the core strength training can help basketball players normally give play to the connection role of human body, guide the coordination and stability of derivative movements of players and make the fulcrum function of human body more firm in terms of power generation links. Meanwhile, the core strength training also plays an important role in technical accuracy of basketball players, which can improve the core stability of players, so as to make them have a good performance while completing the high-difficulty and high-strength technical actions. In addition, the high strength, high antagonism and altitude technique in basketball sports should be enhanced by the core strength training. The core strength training is necessary and possible due to the versatile techniques and tactics and also important due to the flexibility of basketball players.

(3) Injury prevention of basketball players. In basketball sports, the basketball players easily suffer from body injury in the implementation of some basketball techniques and tactics due to the high strength and high antagonism of competition and training. The core strength training can help plays make good use of their core muscles to control the limbs in a normal and reasonable position. We can protect the joints of players and some of their parts that are easily injured. The core strength can reduce or prevent the injury ratio in the physical confrontation or collision in case of any emergency on the basketball court. In addition, the core strength can also help basketball players control their body and keep their body balanced in fierce competition or training. Only in this way, the core strength training can really make basketball players in a beneficial physical state and prevent various injuries in fierce basketball competition or training because it can strengthen the muscle contraction function, improve the power transmission ability and reduce the load and strength of joints in sports. These effective injury prevention will be beneficial to extend the sport life of basketball players.

\section{Practical way of core strength training in basketball sports}

As the core strength training in basketball sports has such important functions and roles, how we conduct the core strength training of basketball players? The specific training practical ways are as follows:

(1) Single unarmed training. In the core strength training of basketball players in basketball sports, the players should be of single unarmed training in the initial stage, which is a training process from outside to inside, from shallow to deep and from slow to quick, with various forms, and can help basketball players stimulate their deep muscles to the greatest extent to generate certain core strength and deeply experience the importance and necessity of core muscles in body 
control. Although it is not generally accepted, it obtains the consent of most experts and scholars. Many scholars think this training method can greatly strengthen the core strength ability of basketball players. In the core strength training, the unarmed training often adopts the basic training method, such as straight back support in side lying, back lying and front lying, in which the players are required to always keep their back fixed, and properly use the power of their waist, and maintain uniform breathing to improve the effect of core strength training. Of course, the core strength training load of players can be increased appropriately with the advancement of the training process.

(2) Training with simple instruments. In basketball sports, the body balance of basketball players and the stability of their technical actions require that the core part of their body does a good job in terms of the stability and control, which requires to conduct strength training with some simple sports equipment in the process of core strength training of basketball players. For example, the roman chair, balance ball and other equipment can be used to exercise the muscle strength of the core regions of players and let the players better grasp the body control method to maintain the correction motion posture in the process of training. It can help basketball players overcome the problem concerning the dependence of the external force in the traditional strength training by virtue of their muscle strength. Of course, in the core strength training of basketball players, the these simple equipment can be combined. It is only required that the players should be of combined multi-equipment core strength training on the basis of certain unarmed or single equipment training, which can effectively increase the training difficulty and also improve their core strength.

(3) Weight training based on the unbalanced state. In basketball sports, the basketball players can also have mechanical weight training. Of course, while increasing the training load of players, attention should be paid to the load, which cannot be too big; otherwise, it will cause unpredictable consequences. Therefore, in the core strength training for basketball players, with increasing the core strength of players as the fundamental purpose, we should adopt the suitable weight training method to make players have effective weight training under the unbalanced state. For example, the players can be of supine weight training for athletes, in which they are required to put the lumbar spine in the middle of the uplifted part of BOSU ball, then lift their legs, and keep the bending degree of knee at 90 degrees. Of course, they can also have double load strength training, in which under the unbalanced state, two players are required to put their hands of different part together, and then wrestle by doing half squat and holding the hand of each other to let a person fall off from the training equipment finally. This double core strength training under the unbalanced state can greatly improve the body control ability and overall power generation of players. In addition, in the core strength training, the basketball players should have many small-load training, either static or dynamic training, which needs to fix the training time and strictly control the players' body posture to improve the training effect.

\section{Notes of core strength training in basketball sports}

The core strength training of basketball players in basketball sports is of huge advantages, but it is not perfect, in which the trainer is required to pay attention to the following matters in the training of players:

(1) Problem concerning training load intensity. The core strength training of basketball players must fully consider the actual training load intensity that the players can bear, in which the players should have weight training according to their gender, age and degree of training, the core strength training should be targeted to avoid causing any damage to their body due to excessive weight, and the training schedule should be well controlled in due order to gradually increase the training load and intensity of players;

(2) Problem concerning systematic training. The core strength training of basketball players should be systematic, which requires us to make them set up long-term and sustained training perseverance in the systematic training arrangement, rather than interrupt in case of any difficulties or other obstacles. In addition, we should listen to their training feedback opinions in the training 
process and then make correction or perfection immediately to achieve the best training effect;

(3) Problem concerning the similarities and differences between the core strength training and the traditional strength training. In the core strength training of basketball players, it is required to find the relationship and difference between the two, rather than completely deny the traditional strength training method, and then coordinate wit the two training methods and highlight the overall function of core strength training. It is also required to increase the height of basketball players in the core strength training platform and carry out the special and diversified core strength training as well as the power transmission and combination training of the core areas of players.

\section{References:}

[1] Huang Ji, Zhao Siqing. Essence of Core Strength Training and Its Practice in Chinese Competitive Sports[J]. Journal of Physical Education, 2010,17(5):74-76.

[2] Jin Qiang. Empirical Research on Influence of Core Stability Training on Flexible Quality of Basketball Players[D]. Beijing Sport University, 2011.

[3] Wang Yajun. Discussion on Definition and Action Mechanism of Core Strength[J]. Journal of Beijing Sport University, 2010.

[4] Yu Cong, Wang Jie. Importance of Core Strength Training in Basketball Training Practice of Colleges[J]. KSZK, 2009(5):161. 